\title{
Analysis of Organic Components by DLLME Based on the Solidification of a Floating Organic Droplet Followed with GC/MS in Wood Vinegar
}

\author{
Qiang Sun*, Yuliang Gao, Liyan Wang \\ Agonomy College, Heilongjiang Bayi Agricultural University, Daqing, China
}

Email address:

byndsunqiang@sina.com (Qiang Sun)

${ }^{*}$ Corresponding author

\section{To cite this article:}

Qiang Sun, Liyan Wang, Yuliang Gao. Analysis of Organic Components by DLLME Based on the Solidification of a Floating Organic Droplet Followed with GC/MS in Wood Vinegar. Science Journal of Analytical Chemistry. Vol. 8, No. 1, 2020, pp. 5-11.

doi: $10.11648 /$ j.sjac.20200801.12

Received: December 21, 2019; Accepted: January 8, 2020; Published: January 31, 2020

\begin{abstract}
A simple, efficient and environmentally friendly method has been established for the analysis of organic components in wood vinegar (WV) by dispersive liquid-liquid micro-extraction based on solidification of floating organic droplet (DLLME-SFO) coupled with GC/MS. Several variables that affect the extraction efficiency, including the type and volume of the extractant and dispersant, extraction time and the mass fraction of sodium chloride were optimized. Under the optimum conditions, the sample extracted were analyzed by GC/MS. The result shows that, apart from the main component of acetic acid, many kinds of ketone compound, phenol compound, ester compound, aldehyde and alcohol compound exist in the WV. The proposed method has been successfully employed to determine the organic components in the WV.
\end{abstract}

Keywords: Wood Vinegar, DLLME-SFO, GC/MS, Organic Components

\section{Introduction}

With the rapid development of agriculture, agricultural mechanization has won greater popularity around the world and straw of crops has attracted global attention. Straw of crops is discarded in farmland and takes a heavy toll for the spring ploughing next year. However, in most China's rural areas, due to the very low efficiency of straw combustion and backward means of straw combustion, the majority of straw is burned on the spot, which leads to severe environmental pollution and biomass resources waste $[1,2]$. A majority of burning gas has wreaked havoc on the survival of human and animal because of the highly toxic property and even led to smog under special conditions [3]. As a result, treatment of corn straw has escalated into a major concern.

Wood vinegar (WV) is gained by clarifying the liquid product of thecorn straw in the carbonization process that is an acidic reddish-brown aqueous liquid [4, 5]. Such volatile organic matter as the WV is produced during the carbonization of the corn straw and is cooled by the smoke window. In agriculture, WV which can be used as fertilizer and a structure-improving agent for the soil contains a variety of organic compounds aimed to promote the growth of plants [6-11]. Until now, lots of products derived from WV have been applied to a wide variety of fields [12-16]. With such products, the gas generated from the combustion of straw can be lessened and the ambient air quality can be improved. The development of green agriculture will be attributed to the application of WV in agriculture.

There are many reports about the application of $\mathrm{WV}$ to various fields, while few analysis about the composition of $\mathrm{WV}$ is reported. However, some researchers can only obtain the organic components in the WV directly from the charcoal kiln or companies specializing in WV in China. The WV is produced from crop straw, birch, pine, apple tree and pear during the carbonization process. Therefore, it's urgent to put forward the methods intended for analysis of the organic components in WV.

Few methods which includes GC [17], HPLC [18], and GC-MS [19] have been raised for the analysis of the components in WV. Even though these analytical methods have been proposed to analyze the components, the 
procedures for sample pretreatment have played an essential role in obtaining sensitive and accurate results. Many methods such as liquid-liquid extraction (LLE) are applied to the pretreatment of the analyzing sample with organic reagents. But LLE has disadvantages because this method is time-consuming and requires large volumes of samples and toxic organic solvents. As a result, more methods have been optimized with the aid of simplified and minimized organic solvents. A new method called DLLME-SFO was introduced by Huang [20, 21] and Feng [22]. As to this method, the analytes are extracted into a small volume of organic solvent in the sample and are further separated by centrifugation. Later, the sample vial is placed into an ice bath or freezing centrifuge and the floated extractant droplet can be collected easily after it is solidified at low temperature. DLLME-SFO has such merits as simple operation, rapidity, low cost, high recovery, and lower toxicity [23-28].

In the paper, DLLME-SFO followed by GC/MS was used for the analysis of the organic components in WV. Different experimental parameters like the type and volume of the extraction and dispersing solvent, extraction time and mass fraction of salt that affect the DLLME-SFO conditions for analysis of organic components of were investigated and optimized in WV. The result reveals that the proposed method is simple, efficient, practical, and environmentally friendly.

\section{Experimental}

\subsection{Instrumentation}

Chromatographic analysis was performed on GC-MS (QP2010, Shimadzu Co., Japan) equipped with EI ion sources. Centrifuge (Z36HK, HERNIE Co., Germany), vortex agitator (VORTEX-2, SI DIGTAL Co., USA), ultrasonic cleaners (SK9500H, Shanghai Yiheng Ultrasonic Instrument Co., Ltd., China) were used for sample preparation.

\subsection{Reagents and Materials}

1-dodecanol, 1-undecanol and n-hexadecane were used as extractant from Sigma Reagent Shanghai Co., Ltd (Shanghai China). Methanol, acetone and ethanol (HPLC grade) were used as dispersant from Aladdin Reagent Shanghai Co., Ltd (Shanghai China). Sodium chloride was calcined at $140^{\circ} \mathrm{C}$ for $4 \mathrm{~h}$ before it can be used, WV was purchased from Dongying RunYiBiotech Co., Ltd. (Dongying, China). $0.22 \mu \mathrm{m}$ microporous membrane was obtained from Bonna-Agela Technologies (Tanjin, China). Other reagents were used at least of analytical reagent grade.

\subsection{GC/MS Analysis}

The sample was analyzed by QP2010 GC/MS. The separation was carried out on RTX-WAX capillary column (30 $\mathrm{m} \times 250 \mu \mathrm{m}$ i.d., film thickness $250 \mu \mathrm{m})$. with the following instrumental conditions: Helium (99.999\%) was as the carrier gas, total flow $25 \mathrm{~mL}$ min-1, column flow $1.3 \mathrm{~mL} \mathrm{~min}-1$, injector temperature $200^{\circ} \mathrm{C}$; transfer line temperature $280^{\circ} \mathrm{C}$; energy of electron $70 \mathrm{eV}$. The oven temperature was programmed as follows: initial temperature $50^{\circ} \mathrm{C}$ (held 10 min), then to $150^{\circ} \mathrm{C}$ at a rate of $10^{\circ} \mathrm{C} \mathrm{min}-1$ and held $10 \mathrm{~min}$, from 150 to $200^{\circ} \mathrm{C}$ at ramp rate of $10^{\circ} \mathrm{C} / \mathrm{min}$ and held $10 \mathrm{~min}$, $200^{\circ} \mathrm{C}$ for $30 \mathrm{~min}$; the injection mode was splitless injecting samples, the quantity of sampling was 1 microlitre.

\subsection{Sample Preparation}

WV through micro-aperturefilter membrane, $2 \mathrm{~mL}$ sample was further transferred into $5 \mathrm{~mL}$ Teflon, $50 \mu \mathrm{L}$ 1-dodecanol (extraction solvent), $800 \mu \mathrm{L}$ ethanol (dispersing solvent), 10\% $(\mathrm{w} / \mathrm{w})$ sodium chloride and rapidly added into the sample solution, and then the mixture was vortexed for 3 min at $-5^{\circ} \mathrm{C}$ centrifugal. After the liquid organic drop had been frozen, water phase was removed with one-off injectors and liquid organic drop was placed in $250 \mu \mathrm{L}$ sample bottle with intubation at room temperature. The extractant was injected into the GC/MS system for the analysis.

\section{Results and Discussion}

In the experiment, micro-aperturefilter membrane $2.0 \mathrm{~mL}$ of WV was used to study the extraction performance under different experimental conditions.

\subsection{Selection of Extraction Solvent}

It is important to choose extractant during the DLLME-SFO process. But the selection of an appropriate solvent is limited by several factors, such as its immiscibility with water, low melting point of $10-30^{\circ} \mathrm{C}$, lower density than water, low volatility, the analytes and the experimental working conditions. After these characteristics are analyzed, the commonly used extraction solvent are 1-undecanol (MP $\left.11^{\circ} \mathrm{C}\right)$, n-hexadecane (MP $18^{\circ} \mathrm{C}$ ) and 1-dodecanol (MP $24^{\circ} \mathrm{C}$ ) in DLLME-SFO which were used to study the extraction of the organic components in $\mathrm{WV}$. However, after freezing, 1-undecanol melts rapidly at room temperature, the peak area of organic components n-hexadecane as extraction solvent is lower than 1-dodecanol (Figure 1). Therefore, 1-dodecanol was adopted for $2.0 \mathrm{~mL}$ supernatant of liquid milk to be the subject in the following analyses. Then further experiments were carried out after 1-dodecanol which was used as the extraction solvent from 2.0 $\mathrm{mL} \mathrm{WV}$ was tested in the subsequent analyses.

To study the effects of the volume of the extractant on the performance of the method, 1-dodecanol of various volumes from 10 to $80 \mu \mathrm{L}$ were investigated in the same DLLME-SFO procedure. The results is presented in Figure 2. It's not easy to collect the extraction solvent from the sample solution for 10 $\mu \mathrm{L}$ 1-dodecanol. The peak area of organic components was successively increased with the volume of 1-dodecanol increased from 20 to $50 \mu \mathrm{L}$. The largest peak area of organic components was $50 \mu \mathrm{L} 1$-dodecanol which was functioned as an extraction solvent for the organic components in $\mathrm{WV}$. Extraction solvent of $50 \mu \mathrm{L}$ tend to make the extraction result keep stable and reach the largest peak area of the organic components. Therefore, $50 \mu \mathrm{L} 1$-dodecanol of extractant was employed in subsequent experiments. 

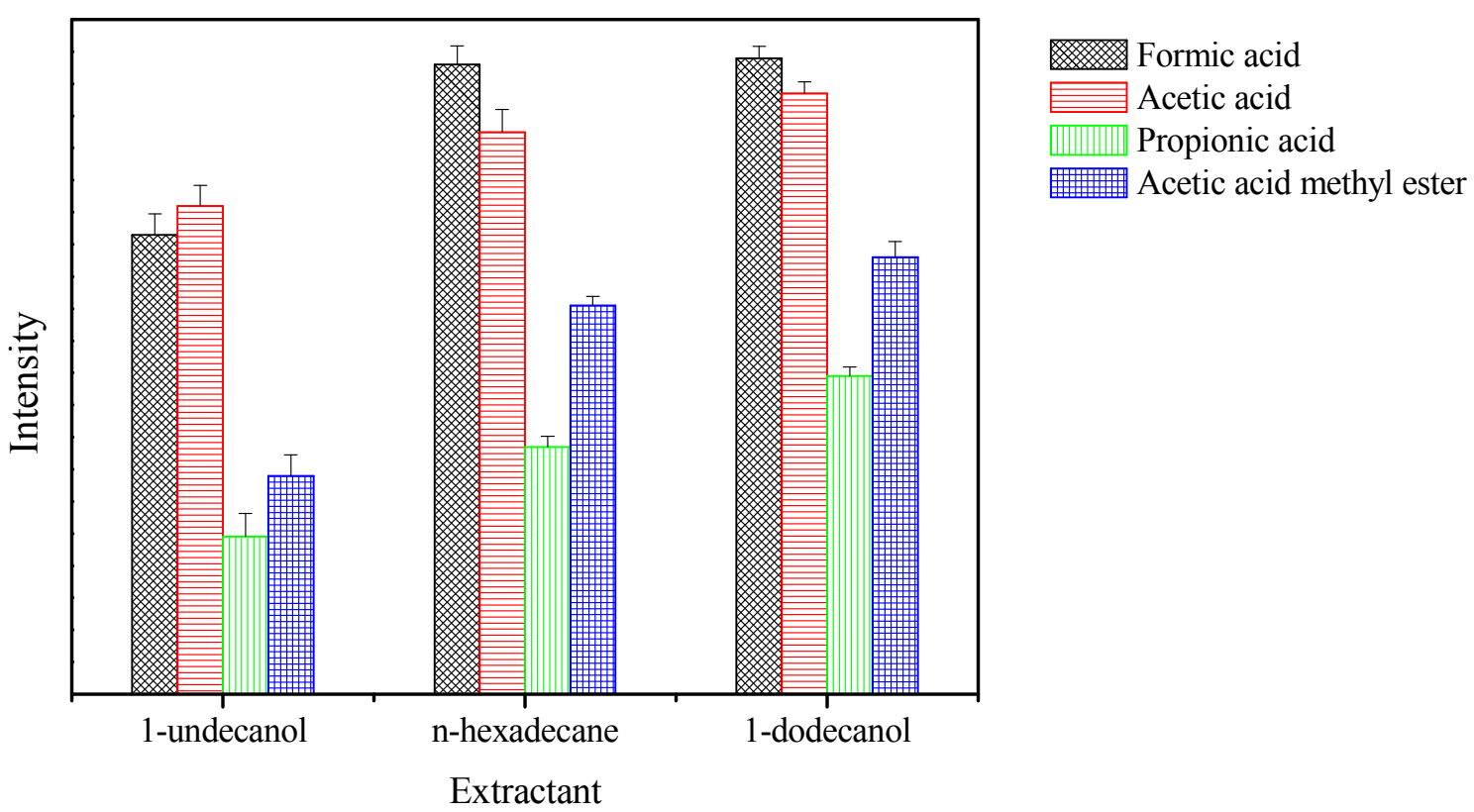

(Note: Intensity of acetic acid methyl ester and propionic acid were amplified 5 times in all figure)

Figure 1. Effect of kind of extractant on intensity.

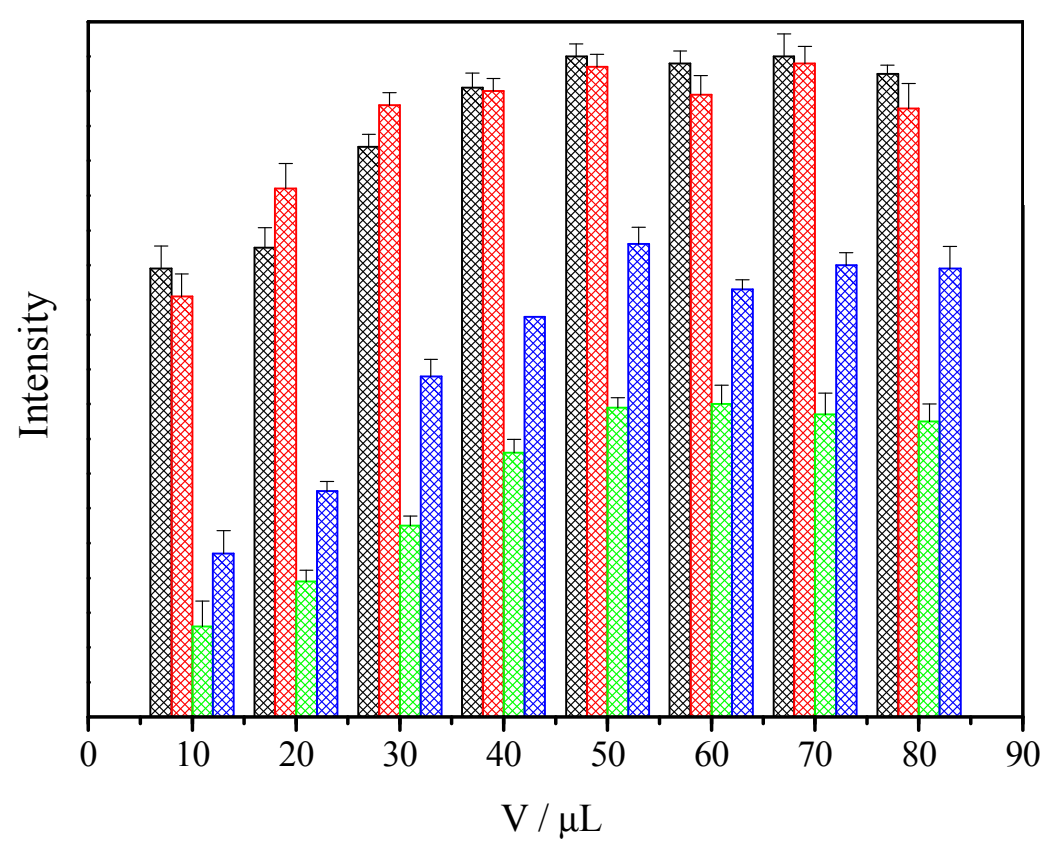

Formic acid

Acetic acid

Propionic acid

Acetic acid methyl ester

Figure 2. Effect of volume of extractant on intensity.

\subsection{Optimization of Type and Volume of Dispersing Solvent}

Dispersing solvent can also play an important role in the extraction performance and composition of analytes. The solubility of analyte decreased with the increase of dispersing volume and reduced extraction efficiency, solvent was not going to work with the decreases of dispersing solvent volume and didn't perform on the cloud system. Methanol, acetone and ethanol as dispersing solvent were studied in Figure 3. Ethanol could be used as dispersing solvent in WV because it had better polarity than the other dispersing solvent and it was better and able to form the cloud system. The influence of the volume of ethanol as dispersing solvent was searched with its volume changed from 100 to 200,400 , $600,800,1000,1200,1400 \mu \mathrm{L}$ respectively (Figure 4). However, it has low extraction efficiency 100 and $400 \mu \mathrm{L}$ of ethanol as dispersing solvent and the stability, reappearing and accuracy of this method are bad. The reason for this could be that, with the ethanol of a lower volumn than 400 $\mu \mathrm{L}$, the extractant could not be dispersed well in the sample solution, thus resulting in a lower peak area. The effect on the extraction efficiency was not evident when the volume of 
ethanol was less than $400 \mu \mathrm{L}$. Extraction efficiency increased with increasing volume of ethanol when it was less than 800 $\mu \mathrm{L}$. When the volume of ethanol reached more than $800 \mu \mathrm{L}$, the peak area decreased. According to these results, $800 \mu \mathrm{L}$ ethanol was selected as the optimum volume of the

\section{dispersing solvent.}

Furthermore, the best extraction efficiency of the target analytes was achieved when 1-dodecanol was used as extraction solvent and ethanol as dispersing solvent.

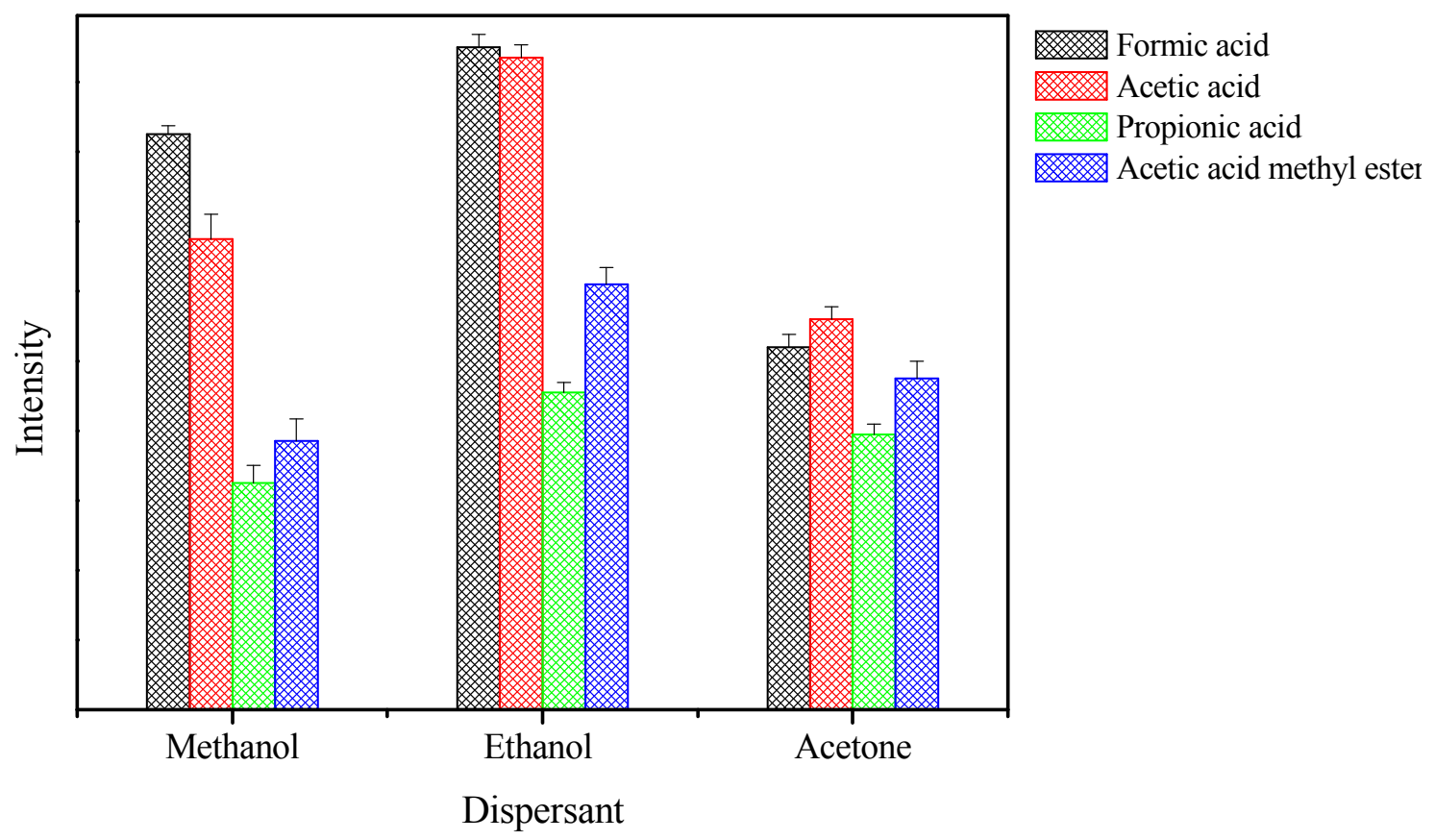

Figure 3. Effect of volume of dispersant on intensity.

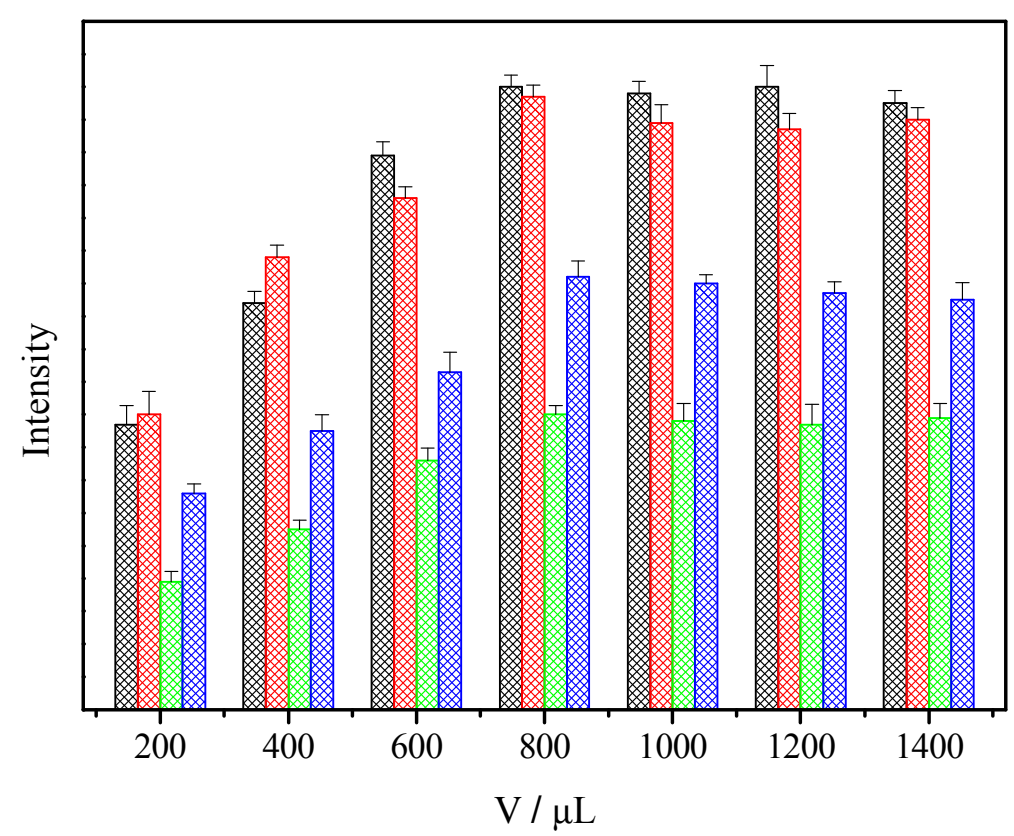

Formic acid

Acetic acid

Propionic acid

Acetic acid methyl ester

Figure 4. Effect of volume of dispersant on intensity.

\subsection{Optimization of Vortex Extraction Time}

It is well known that, in general, the dispersion of the extraction solvent into the sample solution depend on the vortex time. In the study, $50 \mu \mathrm{L}$ 1-dodecanol and $800 \mu \mathrm{L}$ ethanol were put into the sample solution to be oscillated for a period from 1 to $5 \mathrm{~min}$. The results showed that the peak area of the organic components was increased with the extraction time lasting from $1 \mathrm{~min}$ to $3 \mathrm{~min}$, the extraction time increased from 3 to $5 \mathrm{~min}$, and the effect on the peak area of the organic components in WV was not evident. The results revealed that the extraction equilibrium could be achieved when the extraction time lasted for $3 \mathrm{~min}$. Therefore, the optimal extraction time was selected at $3 \mathrm{~min}$. 




Figure 5. Effects of mass fraction of sodium chloride on intensity.

\subsection{Optimization of the Mass Fraction of Sodium Chloride}

The ionic strength of the aqueous phase could be increased to produce more salt in the sample and improved the extraction efficiency of the target compounds. When the concentration of sodium chloride was low, the salting-out played a leading role and the solubility of the target analyte decreased from the aqueous phase to the extraction phase. The extraction efficiency could be enhanced if the dosage of sodium chloride was increased. Density of the aqueous solution was higher than that of the ionic liquid, Thus the recovery of the ionic liquid phase was reduced when the dosage of sodium chloride was added too much. The effect of the adding salt on the extraction efficiency was studied after sodium chloride $0-16 \%(\mathrm{w} / \mathrm{w})$ was added into the sample solution through DLLME-SFO procedure in Figure 5. The experimental results showed that the peak area of the organic components increased with sodium chloride increased from 0 to $10 \%$ in WV. What's more, the peak area of the organic components remained unchanged when the concentration varied in the range of $10-16 \%(\mathrm{w} / \mathrm{w})$. Consequently, $10 \%$ $(\mathrm{w} / \mathrm{w})$ sodium chloride was employed in subsequent experiments.

\subsection{Optimization of Centrifugal Temperature}

The cloudy solution (water, ethanol, and 1-dodecanol) was mixed in $5 \mathrm{~mL}$ centrifuge tubes and the organic components were extracted into the droplets of 1-dodecanol. When the organic liquid drop had been frozen, water phase was removed with one-off injectors and melted liquid organic drop was placed in $250 \mu \mathrm{L}$ sample bottle with intubation at room temperature. The effect of centrifugal temperature on the experiment and the peak area of the organic components was optimized. The experimental results showed that the extraction solvent and WV sample solution would be frozen at low temperature for long time, and then the extraction solvent was easily broken when separated from WV sample solution at high temperature, so $-5^{\circ} \mathrm{C}$ centrifugal for $3 \mathrm{~min}$ was selected in the study.

Table 1. The organic components and relative content of $W V$.

\begin{tabular}{llllll}
\hline Number & Compound & Formula & Molecular weight & Relative contents/\% & Similarity/\% \\
\hline 1 & Formic acid & $\mathrm{CH}_{2} \mathrm{O}_{2}$ & 46 & 4.23 & 98 \\
2 & Acetic acid & $\mathrm{C}_{2} \mathrm{H}_{4} \mathrm{O}_{2}$ & 60 & 4.23 & 95 \\
3 & Ethylene glycol & $\mathrm{C}_{2} \mathrm{H}_{6} \mathrm{O}_{2}$ & 62 & 0.10 & 92 \\
4 & Acetone & $\mathrm{C}_{3} \mathrm{H}_{6} \mathrm{O}$ & 58 & 0.19 & 96 \\
5 & Acetic acid methyl ester & $\mathrm{C}_{3} \mathrm{H}_{6} \mathrm{O}_{2}$ & 74 & 0.42 & 88 \\
6 & Propionic acid & $\mathrm{C}_{3} \mathrm{H}_{6} \mathrm{O}_{2}$ & 74 & 0.68 & 90 \\
7 & $\mathrm{C}_{3} \mathrm{H}_{6} \mathrm{O}_{2}$ & 74 & 0.37 & 94 \\
8 & Hydroxy-acetone & $\mathrm{C}_{4} \mathrm{H}_{6} \mathrm{O}_{2}$ & 86 & 0.28 & 85 \\
9 & 2-butene acid & $\mathrm{C}_{4} \mathrm{H}_{8} \mathrm{O}_{2}$ & 88 & 0.27 & 97 \\
10 & 1-hydroxy-2-butanone & $\mathrm{C}_{4} \mathrm{H}_{8} \mathrm{O}_{2}$ & 88 & 0.28 & 98 \\
11 & Butanoic acid & $\mathrm{C}_{5} \mathrm{H}_{10} \mathrm{O}_{5}$ & 150 & 0.18 & 98 \\
12 & Furfural & $\mathrm{C}_{6} \mathrm{H}_{6} \mathrm{O}$ & 94 & 0.16 & 88 \\
13 & Phenol & $\mathrm{C}_{7} \mathrm{H}_{8} \mathrm{O}_{2}$ & 127 & 0.23 & \\
14 & Phenol, 2methoxy- & $\mathrm{C}_{8} \mathrm{H}_{10} \mathrm{O}_{2}$ & 138 & 0.09 & 90 \\
\hline
\end{tabular}




\section{Real Sample Analysis}

To prove the performance of the present method which was applied to the analysis of real sample, GC/MS method was used to analyse the sample treated by DLLME-SFO. The organic compounds were shown in table 1. The total ion chromatogram of WV was shown in Figure 6. The result showed that besides the main component of acetic acid, many kinds of ketone compound, phenol compound, ester compound, aldehyde and alcohol compound existed in the WV. The proposed method played an important role in the analysis of the organic components in WV.

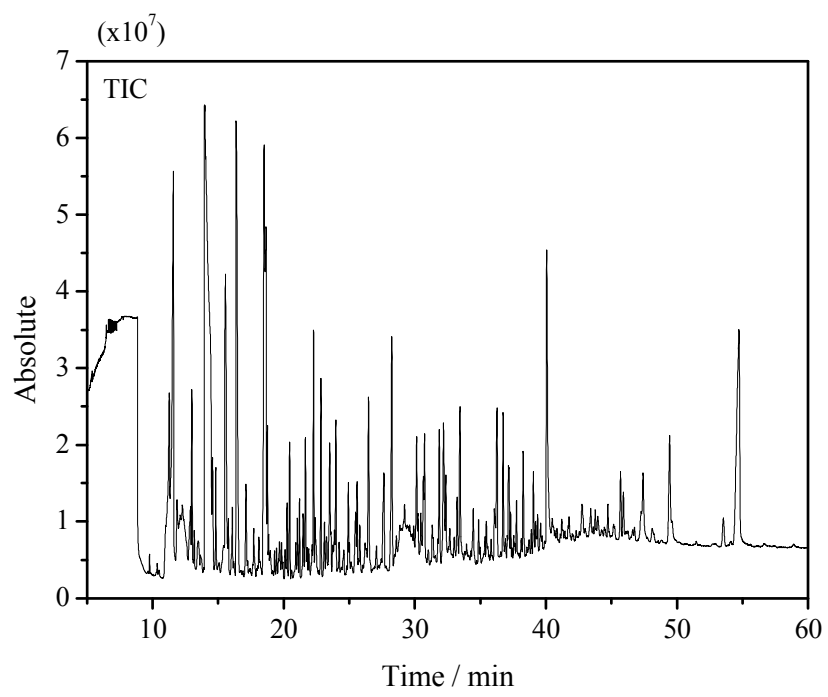

Figure 6. The total ion chromatogram of wood vinegar.

\section{Conclusion}

A method combining DLLME-SFO with GC/MS was developed for the analysis of organic components in WV. For the three extraction solvent applied to the DLLME-SFO procedure, 1-dodecanol displayed especially high efficiency to strengthen the organic components. The organic components are recognized through GC/MS. And the advantages of the proposed method depend on the larger peak area, shorter extraction time, less consumption of solvent, and procedure for the analysis of organic components in WV. In addition, the merits also includes the easier operation, efficiency, simplicity and it is a valuable and an environmentally friendly method.

\section{Acknowledgements}

This work was supported by Center of Management of Laboratory Equipment of Heilongjiang Bayi Agricultural University.

\section{References}

[1] Q. Q. Ren, C. S. Zhao, L. B. Duan and X. P. Chen (2011) NO formation during agricultural straw combustion. Bioresource Technol 102: 7211-7217.
[2] Q. Wei, X. H. Ma and Dong J. N (2010) Preparation, chemical constituents and antimicrobial activity of pyroligneous acids from walnut tree branches. Journal of Analytical and Applied Pyrolysis. J Anal Appl Pyrol 87 (1): 24-28.

[3] A. T.. Arif, C. Maschowski and P. Garra (2017) Cytotoxic and genotoxic responses of human lung cells to combustion smoke particles of Miscanthus straw, softwood and beech wood chips. Atmos Environ 163: 138-154.

[4] Q. M. Wu, S. Y. Zhang and B. X. Hou (2015) Study on the preparation of wood vinegar from biomass residues by carbonization process. Bioresource Technol 179: 98-103.

[5] Y. Baimark, N. Niamsa (2009) Study on wood vinegars for use as coagulating and antifungal agents on the production of natural rubber sheets. Biomass and Bioenerg 33: 994-998.

[6] Y. J. Huo, Z. X. Liu and H. Xuan (2016) Effects of bamboo vinegar powder on growth performance and mRNA expression levels of interleukin-10, interleukin-22, and interleukin-25 in immune organs of weaned piglets. Anim Nutr 2: 111-118.

[7] T. Mungkunkamchao, T. Kesmala and S. Pimratch (2013) Wood vinegar and fermented bioextracts: Natural products to enhance growth and yield of tomato (Solanum lycopersicum L.). Sci Hortic-Amsterdam 154: 66-72.

[8] H. Yoshimura, H. Washio and S. Yoshida (1995) Promoting effect of wood vinegar compounds on fruit-body formation of Pleurotus ostreatus. Mycoscience 36: 173-177.

[9] H. Zheng, C. Z. Sun and X. D. Hou (2018) Pyrolysis of Arundo donax L. to produce pyrolytic vinegar and its effect on the growth of dinoflagellate Karenia brevis. Bioresource Technol 247: 273-281.

[10] H. F. Lu, M. S. Lashari and X. Y. Liu (2015) Changes in soil microbial community structure and enzyme activity with amendment of biochar-manure compost and pyroligneous solution in a saline soil from Central China. Eur J Soil Biol 70: 67-76.

[11] M. S. Lashari, Y. M. Liu and L. Q. Li (2013) Effects of amendment of biochar-manure compost in conjunction with pyroligneous solution on soil quality and wheat yield of a salt-stressed cropland from Central China Great Plain. Field Crop Res 144: 113-118.

[12] D. Angin (2013) Effect of pyrolysis temperature and heating rate on biochar obtained from pyrolysis of safflower seed press cake. Bioresource Technol 128: 593-597.

[13] M. Hagner, O. P. Penttinen and K. Tiilikkala (2013) The effects of biochar, wood vinegar and plants on glyphosate leaching and degradation. Eur J Soil Biol 58: 1-7.

[14] Y. S. Choi, B. J. Ahn and G. H. Kim (2012) Extraction of chromium, copper, and arsenic from CCA-treated wood by using wood vinegar. Bioresource Technol 120: 328-331.

[15] K. S. Shiny, O. K. Remadevi (2014) Evaluation of termiticidal activity of coconut shell oil and its comparison to commercial wood preservatives. Eur J Wood Wood Prod 72: 139-141.

[16] S. Y. Wu, Y. Q. Wu and J. S. Gao (2014) The effect of wood vinegar on hydrothermal liquefaction of cotton stalk under $\mathrm{CO}$ atmosphere. Energ Source Part A 36: 411-417. 
[17] Y. X. Li and H. W. Lin (2012) Comparative study on capillary gas chromatographic analysis of organic components of wood vineger and water-extract of wood-tar prepared form hard wood barks. J anal sci 28: 58-62.

[18] H. Ménard, A. Gaboury and D. Bélanger (1984) High-performance liquid chromatographic analysis of carboxylic acids in pyroligneous liquors. J Anal Appl Pyrol 6: 45-57.

[19] M. Plessi, D. Bertelli and F. Miglietta (2006) Extraction and identification by GC-MS of phenolic acids in traditional balsamic vinegar from Modena. J Food Compos Anal 19: 49-54.

[20] M. I. Leong and S. D. Huang (2008) Dispersive liquid-liquid microextraction method based on solidification of floating organic drop combined with gas chromatography with electron-capture or mass spectrometry detection. J. Chromatogr. A 1211: 8-12.

[21] M. I. Leong and S. D. Huang (2009) Dispersive liquid-liquid microextraction method based on solidification of floating organic drop for extraction of organochlorine fungicides in water samples. J. Chromatogr. A 1216: 7645-7650.

[22] H. Xu, Z. Q. Ding and L. L. Lv (2009) A novel dispersive liquid-liquid microextraction based on solidification of floating organic droplet method for determination of polycyclic aromatic hydrocarbons in aqueous samples. Anal. Chim. Acta 636: $28-33$

[23] Y. L. Gao, X. J. Guo and P. Sun (2017) Ultrasound-assisted
Dispersive Liquid-liquid Microextraction Based on the Solidification of a Floating Organic Droplet Followed by Gas Chromatography for the Determination of Five Pyrethroid Pesticides Residue in Tea Sample. J Tea Sci 37: 527-533.

[24] P. Sun, Y. J. Gao and J. M. Wang (2016) Dispersive liquid-liquid microextraction based on the solidification of a floating organic droplet followed by gas chromatography for the determination of five pyrethroid pesticides residue in liquid milk sample. Chinese J Pestic Sci 18: 497-502.

[25] P. Sun, Y. L. Gao and J. M. Wang (2017) Determination of eugenol residue in aquatic using dispersive liquid-liquid microextraction based on the solidification of a floating organic droplet followed by UHPLC-MS/MS. J. Chin. Mass Spectrom. Soc. 38: 342-3428.

[26] F. R Mansour and N. D. Danielson (2017) Danielson. Solidification of floating organic droplet in dispersive liquid-liquid microextraction as a green analytical tool. Talanta 170: 22-35.

[27] R. Canales, M. Guiñez and S. Cerutti (2017) Determining heterocyclic aromatic amines in aqueous samples: A novel dispersive liquid-liquid micro-extraction method based on solidification of floating organic drop and ultrasound assisted back extraction followed by UPLC-MS/MS. Talanta 174: 548-555.

[28] X. C. Wang, B. Shu and B. Qiu (2017) QuEChERS followed by dispersive liquid-liquid microextraction based on solidification of floating organic droplet method for organochlorine pesticides analysis in fish. Talanta 162: 90-97. 